\title{
Psychosocial, Cognitive, and Physical Impact of Elaborate Consultations and Life Review in Female Patients with Non-Metastasized Breast Cancer
}

\author{
Anja Thronicke ${ }^{a}$ Matthias Kröz ${ }^{a-d}$ \\ Friedemann Schad ${ }^{\mathrm{a}, \mathrm{b}}$ \\ Antje Merkle ${ }^{b} \quad$ Harald Matthes ${ }^{a, b}$ \\ ${ }^{a}$ Research Institute Havelhöhe, Berlin, Germany; \\ ${ }^{b}$ Hospital Havelhöhe, Berlin, Germany; \\ ${ }^{c}$ Charité - Universitätsmedizin Berlin, corporate member of Freie Universität Berlin, Humboldt-Universität zu Berlin, \\ and Berlin Institute of Health, Institute for Social Medicine, Epidemiology and Health Economics, Berlin, Germany; \\ dInstitute for Integrative Medicine, University of Witten/Herdecke, Witten/Herdecke, Germany
}

Cornelia Herbstreit ${ }^{b}$

\section{Keywords}

Breast cancer - Non-metastasized . Health-related quality of life .

Elaborate consultations and life review · Integrative oncology

\section{Summary}

Background: Elaborate consultations and life review (ECLR) has been reg ularly applied in patients of various cancer entities and stages within Anthroposophic-integrative oncology concepts. However, a lack of systematic research in this field has been detected. To close this gap of knowledge, we evaluated the impact of ECLR in patients with non-metastasized breast cancer before, during, and after primary oncological treatment. Methods: Patient-reported outcome measures were evaluated by analyz ing European Organisation for Research and Treatment of Cancer Quality of Life Questionnaires (EORTC QLQ-C30) in patients with non-metastasized breast cancer who had received either oncological standard therapy alone or in combination with ECLR. Results: 95 female patients were eligible for questionnaire analysis (median age 58 years). Adjusted multivariable linear regression analysis revealed that ECLR was associated with significant improvements in medium-term global health/quality of life and emotional, social, and cognitive functioning. Furthermore, ECLR was associated with significant reductions of short-term appetite loss burden, pain, and shortand medium-term financial difficulties. Subgroup analyses revealed significant improvements in pivotal quality-of-life aspects including fatigue $(p=0.002)$ in chemotherapy-treated patients after ECLR. Conclusions: 12 months of surveillance of breast cancer patients reveals medium-term recovery of the global health status/quality of life and cognitive and psychosocial well-being associated with ECLR. In addition, our data indicate a possible association between ECLR and reduced short-term fatigue burden, which has to be re-confirmed prospectively in a larger study cohort. As long-term cancer survivors develop psychological symptoms similar to patients with chronic diseases, prospective studies should evaluate the impact of ECLR on the psychosocial well-being in these patients.

(c) 2018 The Author(s). Published by S. Karger GmbH, Freiburg

\section{Schlüsselwörter}

Brustkrebs · Nicht metastasiert · Gesundheitsbezogene Lebensqualität . Ausführliches ärztliches Beratungs- und Biografiegespräch . Integrative Onkologie

\section{Zusammenfassung}

Hintergrund: Das ausführliche ärztliche Beratungs- und Biografiegespräch (elaborate consultations and life review; ECLR) ist Teil des anthroposophischen und integrativ-onkologischen Behandlungskonzeptes und wird regelmäßig bei Patienten verschiedener Krebsentitäten und -stadien angewendet. Für diese Intervention gibt es derzeit keine systematisch erforschten Daten. Die vorliegende Studie, die den Einfluss des ECLR auf die Lebensqualität von nicht metastasierten Brustkrebspatientinnen evaluiert, soll dazu beitragen, diese Wissenslücke zu schließen. Methoden: Patienten-berichtete Resultate wurden mithilfe von European Organisation for Research and Treatment of Cancer Quality of Life(EORTC QLQ-C30)-Fragebögen ausgewertet, die von Patientinnen ausgefüllt worden waren, die eine onkologische Standardtherapie mit und ohne ECLR erhalten hatten. Die Assoziation der Lebensqualität mit der Intervention wurde nach Adjustierung demografischer und klinischer Variablen in einer multivariablen Analyse vorgenommen. Ergebnisse: 95 weibliche Patienten kamen für die Auswertung der Fragebögen infrage, ihr medianes Alter bei Diagnosestellung betrug 58 Jahre. Eine adjustierte multivariable lineare Regressionsanalyse zeigte, dass das ECLR mit einer signifikanten Verbesserung der mittelfristigen Gesundheit/Lebensqualität sowie emotionaler, sozialer und kognitiver Funktionen assoziiert war. Zudem war das ECLR mit reduzierter Appetitlosigkeit und verringertem Schmerz (6 Monate nach Erstdiagnose) und einer kurz- bis mittelfristigen Reduktion finanzieller Schwierigkeiten assoziiert. Eine Subgruppenanalyse wies signifikante Verbesserungen in zentralen Aspekten der Lebensqualität einschließlich der Fatigue ( $p=$ 0,002 ) nach ECLR-Intervention bei den Patientinnen auf, die eine Chemotherapie erhielten. Schlussfolgerungen: Die Ergebnisse der Nachbeobachtung von Brustkrebspatientinnen zeigten eine mittelfristige (12 Monate nach Erstdiagnose) Verbesserung ihres selbstbewerteten Gesundheitsund Lebensqualitätszustandes sowie ihres kognitiven und psychosozialen Wohlbefindens durch ECLR-Intervention. Zusätzlich weisen unsere Daten auf einen möglichen Zusammenhang zwischen dem ECLR und einer reduzierten kurzfristigen (6 Monate nach Erstdiagnose) Fatigue hin, der jedoch in prospektiven größeren Studien bestätigt werden muss. Da Langzeitüberlebende ähnliche psychologische Symptome entwickeln wie Menschen mit chronischen Erkrankungen, sollte ein Ziel von prospektiven Studien sein, den Einfluss von ECLR auf das psychosoziale Wohlergehen dieser Patienten zu überprüfen.

\section{KARGER}

Fax +497614520714 Information@Karger.com www.karger.com

2018 The Author(s)
Published by S. Karger GmbH, Freiburg
Open acer

This article is licensed under the Creative Commons AttributionNonCommercial-NoDerivatives 4.0 International License (CC BY-NCND 4.0) (http://www.karger.com/Services/OpenAccessLicense). Usage modifed matein requires witten permission.
Dr. med. Friedemann Schad

Hospital Havelhöhe

Kladower Damm 221, 14089 Berlin, Germany

fschad@havelhoehe.de 


\section{Introduction}

Life review (LR) is a natural spontaneous process of convalescence and, as defined by Robert Butler in the 1970's, 'a personal process by which a person evaluates his or her life as it nears its end' [1-5]. Mainly applied in geriatric and palliative care, it helps the person in coping with the disease and to achieve resolution, assurance, internal growth, and reconciliation with family and friends. The combination of elaborate consultations and LR (ECLR) has always been part of medical concepts in Anthroposophic Medicine. Since decades, it has been applied in Anthroposophic-integrative healthcare institutions and evaluates, besides conflicts and issues of the past, perspective-gaining and development principles of the individual human being [6-8].

ECLR becomes visible as retrospection in older age or at earlier time points, when a person experiences major crises or is confronted with death. Facing the diagnosis of cancer can lead into an existential crisis, with feelings of loss of reality, meaning, and security, accompanied by existential fear and despair [9]. In the certified breast cancer center (BCC) of the Gemeinschaftskrankenhaus Havelhöhe [10] specialized in Anthroposophic-integrative oncology, ECLR is applied apart from regular or daily ward rounds and outside of patient rooms, by consciously giving time and space in extra interview appointments. Medical-related questions, e.g. on histology, tumor diagnosis, current therapy, and prognosis, are thoroughly discussed and psychosocial issues such as the place the disease takes in a patient's biography, the patient's personal concept of the disease, or which perspectives of meaning, life and spirituality may be opened up by the disease, are taken into account. The patient's current life situation and personal aims as well as social and familial relationships and communication (problems) are illuminated. Furthermore, the patient's emotional status is looked at. Question are raised as to how much the patients respect their needs and demands, what kind of guidance for coping with the disease is available to the patients, and what seems to be important in the light of the disease. Finally, post-hospital issues such as outpatient psycho-oncological therapy, support groups, and medical support may as well be part of the ECLR. So far, systematic research in oncology has been made only in terminally ill cancer patients to evaluate patient-reported outcomes after an LR. The results of these studies indicate that the LR improved spiritual well-being and psychosocial distress [11-13]. No data exist for the impact of ECLR on non-metastasized breast cancer patients or survivors.

Breast cancer is the most common cancer among young adults ( $<45$ years, incidence: 61 per 100,000 women) and among adults aged 45-64 years (531 per 100,000 women) in Europe [14]. The prevalence rate is increasing due to the diffusion of screening methods and the improvement of survival rates. Long-term survivors may develop symptoms comparable to those observed in chronic illnesses. As shown by Elliott et al. [15] in the supplement of the National Cancer Survivorship Initiative vision report, longterm survivors had poorer health and well-being compared to people without cancer. As a consequence, a five-shift proposal in care and support for long-term survivors has been introduced to un- ravel and satisfy unmet needs of cancer survivors [16]. Besides the suggestion of improvement of personalized and tailored follow-up care and self-management instead of clinical management, the authors suggest the routine use of patient-reported outcome measures (PROMs) in post-therapy care [16]. Therefore, the study and improvement of health-related quality of life (HRQL) has become increasingly important in comprehensive care and support of breast cancer patients and survivors. It has already been acknowledged that HRQL, physical health, pain, and loss of appetite are prognostic factors in patients with cancer including breast cancer [17] and that HRQL and psychological factors are predictive of the survival of metastasized breast cancer patients $[18,19]$.

The present observational study explored the impact of standard care plus ECLR on PROMs in breast cancer patients before, during, and after therapy, compared to patients having received standard care alone. As indicated by a meta-analysis in 2008, the European Organisation for Research and Treatment of Cancer Quality of Life Questionnaire-C30 (EORTC QLQ-C30) was 1 of the 4 most common and well-developed valid instruments to measure HRQL in breast cancer patients. To supply high quality standards and to make the results comparable [20], data from EORTC QLQ-C30 questionnaires that were answered by patients being treated in a certified German BCC were evaluated in the present study; the BCC is annually audited by the country's largest scientific-oncological medical expert association, the German Cancer Association (DKG) [10].

\section{Material and Methods}

\section{Study Design}

We conducted an observational study for all patients with a primary diagnosis of stage I-III breast cancer seen between January 2011 and June 2016 at the certified BCC at the Gemeinschaftskrankenhaus Havelhöhe, Berlin, Germany who were 18 years or older, who gave written consent, and from whom completed forms of EORTC QLQ-C30 questionnaires at all 3 measured time points (baseline, first diagnosis (T0), 6 months after first diagnosis (T1), and 12 months after first diagnosis (T2)) were available. Exclusion criteria were defined as having a diagnosis of metastatic disease at the time of diagnosis and missing forms (the whole questionnaire is missing for a patient) at any of the 3 measured time points. Furthermore, in the case of missing scale scores, the patient was not eligible for the study. For missing items (1 or more missing answers to questions within the questionnaire), imputation techniques for multiitem scales according to Fayers and Machin [21] were performed. For this technique, at least half of the items from the scale needed to be answered and singleitem measures needed to be complete. Equations were made for multi-item scales equivalent to equations made as described in the EORTC QLQ-C30 manual [22]. The study was approved by the ethical committee of the Medical Association Berlin (Eth-27/10)

\section{Data Collection}

Demographic data as well as information on diagnosis, histology, surgery, and previous treatment regimens were retrieved from the network oncology (NO) registry. NO is an accredited clinical registry of hospitals and out-patient practitioners specialized in Anthroposophic and integrative oncology [23]. The registry utilizes linked data from primary and secondary care in concert with cancer registry data and hospital episode statistics. Retrieved data included application procedures of multimodal integrative therapies (standard care) consisting of a combination of guideline-orientated conventional oncological care 
and add-on complementary therapy with or without ECLR therapy. Effects were evaluated as short-term effects at T1 (6 months after first diagnosis) and medium-term or sustained effects at T2 (12 months after first diagnosis)

\section{Classification of Groups}

ECLR interviews were performed at the beginning and at the end of a patient's hospital stay or, if exceeding 1 week of hospitalization, within the next 7 days. Patients who received ECLR interviews in addition to standard care were allocated to the 'ECLR' group, the other patients who received standard care, to the 'control' group (Ctrl).

\section{Endpoints}

The primary outcome of the study was to investigate differences in patientreported psychological, social, and/or physical outcomes at $\mathrm{T} 1$ and $\mathrm{T} 2$ between patients treated either with or without ECLR concomitantly to standard therapy, in order to test the hypothesis that additional ECLR was associated with short-term (6 months after first diagnosis) and medium-term (12 months after first diagnosis) improvement of pivotal areas of HRQL. The secondary outcome was the explorative evaluation of differences of PROMs in chemotherapytreated patients from the ECLR and control groups.

\section{Statistical Analysis}

For both groups, baseline characteristics and treatment regimens were compared using the Wilcoxon-Mann-Whitney test for categorical and continuous variables. The Wilcoxon-Mann-Whitney test and the 2-sided Student's t-test were performed to detect differences in PROMs between the ECLR and control groups. To quantify the strength of the relationship between PROMs and ECLR or other interventions, a multivariable linear regression analysis was performed. This model was applied for each of the 5 numerical functioning and 9 numerical symptom outcomes at both follow-up time points as response variable. In order to yield reliable model results and to abstain from overfitting, we introduced a regression subset selection (R package 'leaps', version 3.0) [24, 25] including exhaustive search to select for a subset of reliable variables. The model with an indicated number of variables with the highest adjusted $\mathrm{R}^{2}$ was chosen as the best model. Given that all other predictor variables were 'held fixed' (age (in years), Union for International Cancer Control (UICC) stage (categorical, I-III), surgery (yes/no), oncological standard treatment (yes/no; including chemotherapy, radiotherapy, immunological therapy, hormonal therapy), concomitant Viscum album L. (VA) treatment (yes/no), non-pharmacological interventions (yes/no) such as nursing, movement therapies, massages, art therapies, and psycho-oncological support), the relationship between each listed PROM and ECLR was evaluated. Continuous variables were described as median with interquartile range (IQR); categorical variables were summarized as frequencies and percentages. Data distributions were inspected graphically using box plots and histograms and were arithmetically examined for skewness. $\mathrm{p}$-values $<0.05$ were considered to be significant. All statistical analyses were performed using the software $\mathrm{R}$ (version 3.3.0) [26].

\section{Results}

\section{Patient Characteristics}

From 239 women with non-metastasized breast cancer (173 with ECLR and 66 without additional ECLR), complete data were obtained, and all 3 questionnaire forms were collected for 95 eligible patients receiving integrative oncological care with $(\mathrm{n}=31)$ or without ( $\mathrm{n}=64$ ) additional ECLR (see also the study flow chart in fig. 1). Table 1 shows the main characteristics of the patients at baseline. The patients from the ECLR group were in median 54 years old, i.e., 6.5 years younger than the patients from the control group (60.5 years; $\mathrm{p}=0.2)$. Human epidermal growth factor receptor 2-positive (HER2+) disease was documented in 4 (12.9\%)

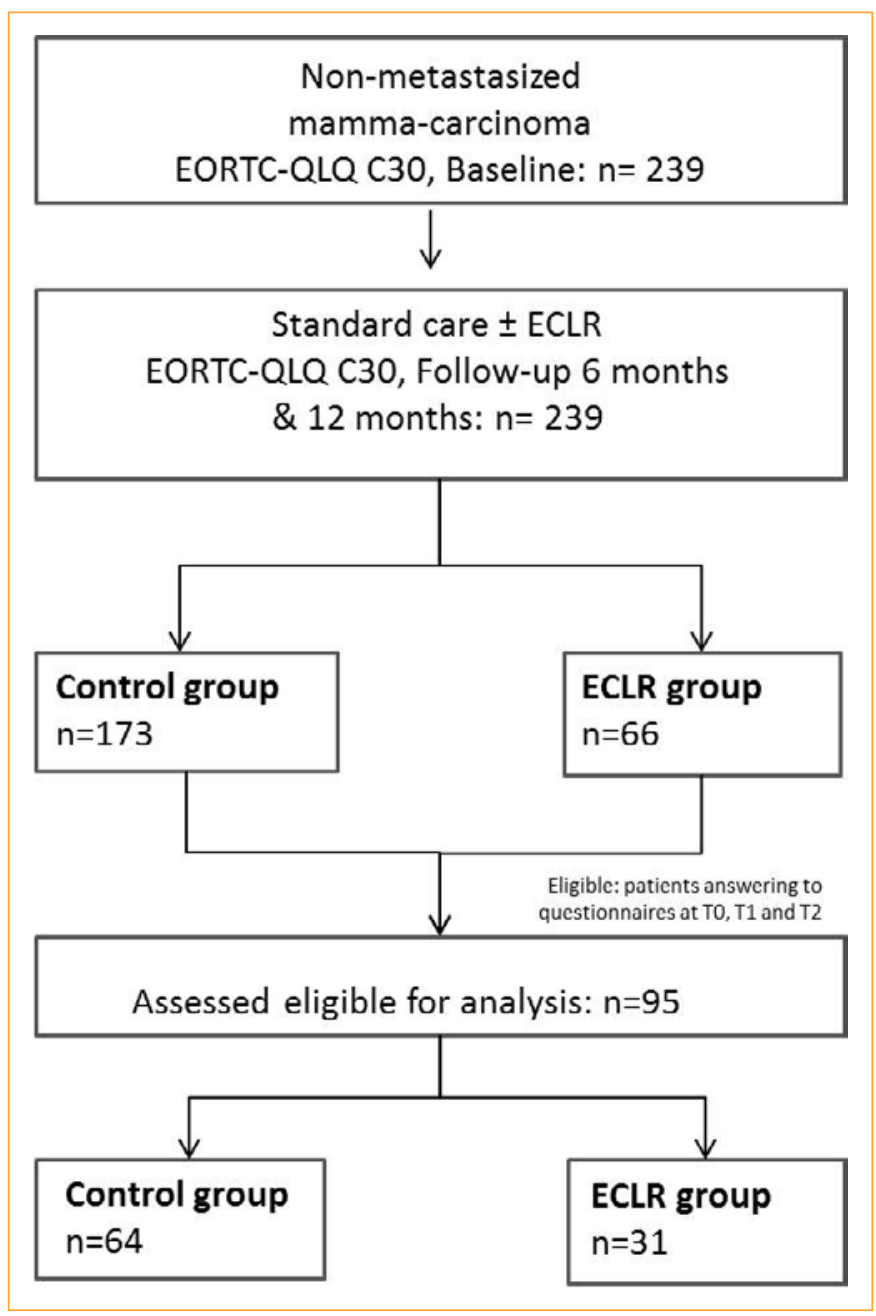

Fig. 1. Flow chart of the study population. Control group: patients treated with guideline-oriented oncological standard therapy; ECLR group: patients treated with a combination of guideline-oriented oncological standard therapy and ECLR. $\mathrm{n}=$ Number of patients, UICC stage IV = metastasized patients (TNM staging according to the Union for International Cancer Control). Excluded patients with missing 6 or 12 months of surveillance (missing forms) at the time of analysis are still under follow-up. ECLR = elaborate consultations and life review; EORTC-QLQ C30 = European Organisation for Research and Treatment of Cancer quality-of-life questionnaire C30.

of the ECLR patients and in $9(14.0 \%)$ patients of the control group. None of the ECLR patients and $3(4.7 \%)$ of the control group patients had HER2+, estrogen receptor-negative (ER-), and progesterone receptor-negative (PR-) tumors. Triple-negative tumors were documented in $8(25.8 \%)$ and $16(25.0 \%)$ of the ECLR and Ctrl patients, respectively. No significant difference between groups with regard to tumor subtypes and other baseline characteristics was found. The baseline EORTC QLQ-C30 results were within the range of formerly published EORTC QLQ-C30 reference values for breast cancer patients (table 2) [27].

\section{Oncological Treatment}

No statistically significant differences were observed regarding integrative oncological treatment between the two groups. As to 
Table 1. Demographic data and characteristics at baseline $\mathrm{e}^{\mathrm{a}}$
Table 2. Summary of questionnaire scores at baseline and comparison with reference values $^{\mathrm{a}}$

\begin{tabular}{|c|c|c|c|}
\hline & Control, n (\%) & ECLR-treated patients, n (\%) & $\mathrm{p}$ \\
\hline Total number of patients & 64 & 31 & \\
\hline Age, years, median (IQR) & $60.5(50.0-68.5)$ & $54.0(49.5-60.5)$ & 0.2 \\
\hline Gender, female & $64(100)$ & $31(100)$ & \\
\hline \multicolumn{4}{|l|}{ Tumor subtypes } \\
\hline HER2 $^{-}$ & $52(81.3)$ & $20(64.5)$ & $0.9^{c}$ \\
\hline $\mathrm{ER}^{+}$or $\mathrm{PR}^{+}$or both, and HER2 ${ }^{-}$ & $35(54.7)$ & $12(38.7)$ & \\
\hline $\mathrm{ER}^{+}$or $\mathrm{PR}^{+}$or both, and HER2-, Ki-67 $\geq 35 \%$ & $3(4.7)$ & $1(3.2)$ & \\
\hline Triple-negative status $\left(\mathrm{ER}^{-}, \mathrm{PR}^{-}, \mathrm{HER} 2^{-}\right)$ & $16(25)$ & $8(25.8)$ & $0.9^{\mathrm{d}}$ \\
\hline $\mathrm{HER}^{+}$ & $9(14.0)$ & $4(12.9)$ & \\
\hline $\mathrm{HER}^{+}, \mathrm{ER}^{-}, \mathrm{PR}^{-}$ & $3(4.7)$ & 0 & \\
\hline \multicolumn{4}{|l|}{ Hormonal status } \\
\hline Postmenopausal & $28(43.8)$ & $9(29.0)$ & \\
\hline \multicolumn{4}{|l|}{ UICC stage ${ }^{\mathrm{b}}$} \\
\hline $0-$ II & $58(90.6)$ & $29(93.5)$ & $0.08^{\mathrm{e}}$ \\
\hline III & $6(9.4)$ & $2(3.1)$ & \\
\hline \multicolumn{4}{|l|}{ Type of mastectomy } \\
\hline Radical & $6(9.4)$ & $5(16.1)$ & 0.3 \\
\hline Segmental & $57(89.1)$ & $28(90.3)$ & 0.9 \\
\hline
\end{tabular}

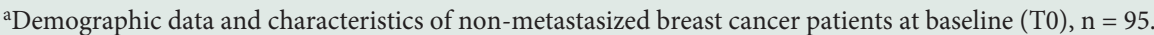

bUICC stages at first diagnosis according to the 7th edition of the 'TNM Classification of Malignant Tumours'.

${ }^{\mathrm{c}}$ Reference $=$ HER2 status

${ }^{\mathrm{d}}$ Reference $=$ triple-negative status

${ }^{\mathrm{e}}$ Reference $=$ stage 0 .

$\mathrm{ECLR}$ = Elaborate consultations and life review; IQR = interquartile range; HER2 = human epidermal growth factor receptor 2; $\mathrm{ER}$ = estrogen receptor; PR = progesterone receptor; UICC = Union for International Cancer Control.

\begin{tabular}{|c|c|c|c|c|c|}
\hline & Key & Mean & SD & IQR & Reference values, IQR \\
\hline Global health status/QoL & QL & 59.84 & 18.75 & $50.0-75.0$ & $50.0-83.3$ \\
\hline Physical functioning & $\mathrm{PF}$ & 78.15 & 18.16 & $67.0-93.0$ & $66.7-93.3$ \\
\hline Role functioning & $\mathrm{RF}$ & 58.23 & 31.91 & $33.0-83.0$ & $50-100.0$ \\
\hline Emotional functioning & $\mathrm{EF}$ & 54.93 & 24.80 & $42.0-75.0$ & $58.3-91.7$ \\
\hline Cognitive functioning & $\mathrm{CF}$ & 74.56 & 24.23 & $67.0-100$ & $66.7-100.0$ \\
\hline Social functioning & SF & 60.55 & 31.34 & $33.0-83.0$ & $66.7-100.0$ \\
\hline Fatigue & FA & 41.84 & 26.84 & $22.0-56.0$ & $11.1-44.4$ \\
\hline Nausea and vomiting & NV & 7.26 & 13.20 & $0-17.0$ & $0-0^{\mathrm{b}}$ \\
\hline Pain & $\mathrm{PA}$ & 31,44 & 24.50 & $0-50.0$ & $0-33.0$ \\
\hline Dyspnea & DY & 18.54 & 27.68 & $0-33.0$ & $0-33.0$ \\
\hline Insomnia & SL & 39.96 & 36.13 & $0-67.0$ & $0-33.0$ \\
\hline Appetite loss & $\mathrm{AP}$ & 20.32 & 29.57 & $0-33.0$ & $0-33.0$ \\
\hline Constipation & $\mathrm{CO}$ & 8.41 & 19.36 & $0-0$ & $0-33.0$ \\
\hline Diarrhea & DI & 8.75 & 20.06 & $0-0$ & $0-0^{c}$ \\
\hline Financial difficulties & FI & 30.13 & 33.58 & $0-50.0$ & $0-33.0$ \\
\hline
\end{tabular}

a Summary of the 15 scale scores of the EORTC QLQ-C30 questionnaire at baseline for non-metastasized breast cancer patients $(\mathrm{n}=95)$ and comparison to EORTC QLQ-C30 reference values for breast cancer, age 50-59 years. Reference values provided by the EORTC Quality of Life Group Members and others of the QLQ-C30 [27].

${ }^{b}$ Mean and SD for reference value: $7.3,16.8$.

${ }^{\mathrm{c}}$ Mean and SD for reference value: $5.5,14.3$.

QoL = Quality of life; SD = standard deviation; IQR = interquartile range; EORTC-QLQ-C30 = European Organisation for Research and Treatment of Cancer quality-of-life questionnaire.

pharmacological treatment, the majority of the patients of both groups received anti-hormonal therapy, more than one-third received mistletoe treatment and one-fifth to one-third received chemotherapy; for details, please see table 3. Only a small propor- tion of patients of both groups received immunological therapy including monoclonal antibodies. Radiation was applied in more than half of patients. Concerning non-pharmacological interventions, no significant differences were seen between the 2 groups. 
Table 3. Oncological treatment $^{\mathrm{a}}$

\begin{tabular}{|c|c|c|c|}
\hline & Control, n (\%) & ECLR-treated patients, n (\%) & $\mathrm{p}$ \\
\hline Total number of patients & 64 & 31 & \\
\hline Chemotherapy & $11(19.3)$ & $9(29.0)$ & 0.9 \\
\hline Radiation & $51(79.7)$ & $22(71.0)$ & 0.3 \\
\hline Whole mamma & $48(75)$ & $21(67.7)$ & \\
\hline Partial mamma & $2(3.1)$ & 0 & \\
\hline Hormonal therapy & $45(70.3)$ & $19(61.3)$ & 0.4 \\
\hline Immunological therapy/mAb & $5(7.8)$ & $1(3.2)$ & 0.4 \\
\hline Viscum album L. therapy & $28(43.8)$ & $13(41.9)$ & 0.9 \\
\hline Nursing & $64(100)$ & $31(100)$ & NA \\
\hline Art therapy & $63(98.4)$ & $29(93.5)$ & 0.2 \\
\hline Movement (eurythmy therapy) & $58(90.6)$ & $28(90.3)$ & 1.0 \\
\hline Psycho-oncological treatment & $58(90.6)$ & $25(80.6)$ & 0.2 \\
\hline Massages & $49(76.6)$ & $20(64.5)$ & 0.2 \\
\hline Breathing therapy & $1(1.6)$ & $1(3.2)$ & 0.2 \\
\hline
\end{tabular}

aPharmacological and non-pharmacological interventions of non-metastasized breast cancer patients $(\mathrm{n}=95)$ treated with standard care without (Control) or with ECLR.

$\mathrm{ECLR}=$ Elaborate consultations and life review; $\mathrm{mAb}=$ monoclonal antibody; $\mathrm{NA}=$ not applicable.

The most applied ones were nursing interventions followed by art therapy, movement therapy, psycho-oncological treatment, and massages.

\section{Differences in Patient-Reported Outcomes between Groups}

Quality of life (QoL) and functioning scores were balanced between both groups at baseline. With the exception of pain ( $\mathrm{p}=$ $0.02)$ and insomnia $(\mathrm{p}=0.03)$, the symptom scores at baseline did not differ between groups. We observed a significant difference between groups in patient-reported T1 pain (ECLR: $19.3 \pm 4.5$, Ctrl: $32.5 \pm 3.9$, difference $-13.2 ; \mathrm{p}<0.05$ ) (table 4). Furthermore, significant differences in financial difficulties at T1 (ECLR: 18.2 \pm 4.0 , Ctrl: $34.3 \pm 5.0$, difference $-16.2 ; \mathrm{p}<0.05$ ) and at T2 (ECLR: $12.8 \pm$ 3.7, Ctrl: $27.6 \pm 4.2$, difference $-14.7 ; \mathrm{p}<0.05$ ) were seen.

The self-reported T2 global health status/QoL (table 5) was significantly improved in the ECLR group $(76.0 \pm 2.8)$ compared to the control $(64.3 \pm 2.5)$, with a clinically relevant difference of 11.7 $(\mathrm{p}<0.01)$. With regard to the functioning scores, highly significant and clinically relevant differences between the two groups were seen in patient-reported T2 emotional functioning (ECLR: $74.7 \pm$ 4.8, Ctrl: $60.8 \pm 3.1$, difference 13.9; $\mathrm{p}<0.01)$ and T2 cognitive functioning (ECLR: $85.4 \pm 3.1$, Ctrl: $71.3 \pm 3.0$, difference 14.4; $\mathrm{p}<$ 0.01 ) (table 5). Also patient-reported social functioning at T1 (ECLR: 18.2 \pm 3.6 , Ctrl: 66.2 \pm 3.8 , difference 12.3; $\mathrm{p}<0.05$ ) and at T2 (ECLR: $80.6 \pm 3.8$, Ctrl: $64.6 \pm 3.9$, difference 16.1; $\mathrm{p}<0.05$ ) was significantly elevated in the ECLR group compared to the control.

\section{Association between Patient-Reported Outcomes and ECLR}

On multivariable analysis adjusted for age, cancer stage, chemotherapy treatment, surgery, radiation, and integrative oncological treatments, ECLR was significantly associated with a reduction of the patient-reported T1 appetite loss $(\beta=-12.5 ; \mathrm{p}=0.03)$, of T1 pain $(\beta=-12.0 ; p=0.04)$, and of reported T1 $(\beta=-19.8 ; p=0.01)$ and T2 financial difficulties $(\beta=-18.0 ; \mathrm{p}=0.004)$ (table 4$)$. Self- judgement of T2 appetite loss and T2 pain was not significantly associated with this intervention.

Furthermore, the analysis showed that ECLR was associated with an improvement of the self-reported T2 global health status/ QoL $(\beta=10.9 ; \mathrm{p}=0.01)$ and $\mathrm{T} 2$ emotional $(\beta=11.9 ; \mathrm{p}=0.03)$, T2 social $(\beta=12.5 ; \mathrm{p}=0.04)$, and T2 cognitive functioning $(\beta=12.6$; $\mathrm{p}=0.01)$.

\section{Association between Patient-Reported Outcomes and Other Prognostic Factors}

We observed that increasing age was highly significantly associated with improvement of financial difficulties at T1 $(\mathrm{p}<0.01)$ and $\mathrm{T} 2(\mathrm{p}<0.01)$ (table 4$)$ and worsening of physical functioning at T1 $(\mathrm{p}<0.01)$ and T2 $(\mathrm{p}<0.01)$ (table 5). Furthermore, UICC stage IIIA was associated with an increase of the self-reported fatigue burden at T1 $(\mathrm{p}<0.05)$ (table 4) and an impairment of emotional functioning $(\mathrm{p}<0.05)$ at T1 (table 5$)$. However, massage was positively associated with emotional functioning at T2 $(\mathrm{p}<0.05)$ (table 5). Mistletoe treatment was significantly associated with a reduction of the appetite loss burden at T2 (p < 0.05) (table 4). Hormonal therapy reduced the diarrhea burden at both time points, T1 and T2 (both $\mathrm{p}<0.05$ ) (table 4). However, immunological therapy was associated with a worsening of the self-reported diarrhea burden at $\mathrm{T} 1(\mathrm{p}<0.01)$.

\section{Association between Patient-Reported Outcomes and Chemotherapy}

A subgroup analysis in 27 patients treated with chemotherapy revealed significant differences in the self-reported fatigue burden between the chemotherapy-treated ECLR and control groups at T1 (fig. 2). Chemotherapy-treated patients who received no additional ECLR reported a significantly higher burden of fatigue compared to patients from the ECLR group (mean score at T1 \pm standard error (SE) for Ctrl: $56.9 \pm 5.7$ and for the ECLR group: $22.1 \pm 6.9$; 
Table 4. Differences in patient-reported outcomes (symptoms) between groups and association with ECLR ${ }^{\mathrm{a}}$

\begin{tabular}{|c|c|c|c|c|}
\hline \multirow[t]{2}{*}{ Symptom } & \multicolumn{2}{|c|}{ Questionnaire score (mean $\pm \mathrm{SE})$} & \multirow{2}{*}{$\begin{array}{l}\text { Difference } \\
\left(\mathrm{p}^{\star}\right)\end{array}$} & \multirow{2}{*}{$\begin{array}{l}\text { Estimate }\left(\beta^{* *}\right) \text { for } \\
\operatorname{ECLR}\left(\mathrm{p}^{* * *}\right)\end{array}$} \\
\hline & $\begin{array}{l}\text { Control } \\
(\mathrm{n}=64)\end{array}$ & $\begin{array}{l}\text { ECLR treatment } \\
\text { group }(\mathrm{n}=31)\end{array}$ & & \\
\hline \multicolumn{5}{|l|}{ Pain } \\
\hline Baseline (T0) & $36.3 \pm 3.5$ & $21.5 \pm 3.5$ & $-14.8(0.02)$ & \\
\hline 0.5 -year follow-up (T1) & $32.5 \pm 3.9$ & $19.3 \pm 4.5$ & $-13.2(0.04)$ & $-12.0(0.05)$ \\
\hline 1-year follow-up (T2) & $26.1 \pm 3.3$ & $15.5 \pm 3.6$ & $-10.5(0.05)$ & $-9.2(0.19)$ \\
\hline \multicolumn{5}{|l|}{ Dyspnea } \\
\hline Baseline (T0) & $21.3 \pm 3.7$ & $12.9 \pm 3.7$ & $-8.4(0.2)$ & \\
\hline 0.5 -year follow-up (T1) & $21.3 \pm 3.7$ & $12.9 \pm 4.0$ & $-8.4(0.2)$ & $-11.4(0.07)$ \\
\hline 1-year follow-up (T2) & $23.9 \pm 3.7$ & $18.2 \pm 4.9$ & $-5.7(0.4)$ & $-1.4(0.83)$ \\
\hline \multicolumn{5}{|l|}{ Insomnia } \\
\hline Baseline (T0) & $45.3 \pm 4.4$ & $28.9 \pm 4.4$ & $-16.4(0.03)$ & \\
\hline 0.5 -year follow-up (T1) & $41.2 \pm 4.5$ & $40.8 \pm 6.1$ & $-0.3(1.0)$ & $-3.9(0.64)$ \\
\hline 1-year follow-up (T2) & $41.6 \pm 4.8$ & $39.8 \pm 6.3$ & $-1.8(0.9)$ & $1.6(0.83)$ \\
\hline \multicolumn{5}{|l|}{ Fatigue } \\
\hline Baseline (T0) & $44.9 \pm 3.4$ & $35.5 \pm 3.4$ & $-9.5(0.1)$ & \\
\hline 0.5 -year follow-up (T1) ${ }^{\mathrm{b}}$ & $41.5 \pm 3.2$ & $35.5 \pm 4.3$ & $-6.0(0.3)$ & $-5.0(0.31)$ \\
\hline 1-year follow-up (T2) & $41.4 \pm 3.3$ & $31.5 \pm 4.1$ & $-9.9(0.1)$ & $-5.4(0.35)$ \\
\hline \multicolumn{5}{|l|}{ Appetite loss } \\
\hline Baseline (T0) & $22.4 \pm 3.8$ & $16.1 \pm 3.8$ & $-6.3(0.3)$ & \\
\hline 0.5 -year follow-up $(\mathrm{T} 1)^{\mathrm{c}}$ & $16.6 \pm 3.5$ & $7.5 \pm 3.7$ & $-9.1(0.1)$ & $-12.5(0.03)$ \\
\hline 1-year follow-up (T2) & $9.3 \pm 2.4$ & $9.7 \pm 4.4$ & $0.3(0.6)$ & $1.7(0.74)$ \\
\hline \multicolumn{5}{|l|}{ Nausea and vomiting } \\
\hline Baseline (T0) & $5.5 \pm 1.4$ & $10.8 \pm 1.4$ & $5.3(0.1)$ & \\
\hline 0.5 -year follow-up (T1) & $5.3 \pm 1.3$ & $7.6 \pm 2.3$ & $2.3(0.4)$ & $3.4(0.26)$ \\
\hline 1-year follow-up (T2) & $2.9 \pm 1.1$ & $2.7 \pm 1.4$ & $-0.2(0.8)$ & $1.8(0.40)$ \\
\hline \multicolumn{5}{|l|}{ Constipation } \\
\hline Baseline (T0) & $8.3 \pm 2.4$ & $8.6 \pm 2.4$ & $0.3(0.8)$ & \\
\hline 0.5-year follow-up (T1) & $14.0 \pm 3.8$ & $5.4 \pm 2.7$ & $-8.7(0.2)$ & $-4.1(0.51)$ \\
\hline 1-year follow-up (T2) & $12.5 \pm 3.4$ & $5.4 \pm 3.5$ & $-7.1(0.1)$ & $-6.3(0.25)$ \\
\hline \multicolumn{5}{|l|}{ Diarrhea } \\
\hline Baseline (T0) & $8.3 \pm 2.3$ & $9.7 \pm 2.3$ & $1.3(0.9)$ & \\
\hline 0.5 -year follow-up $(\mathrm{T} 1)^{\mathrm{d}}$ & $9.3 \pm 2.5$ & $15.0 \pm 3.7$ & $5.6(0.1)$ & $6.28(0.16)$ \\
\hline 1-year follow-up (T2) & $11.4 \pm 2.9$ & $9.6 \pm 3.5$ & $-1.8(0.8)$ & $-3.08(0.55)$ \\
\hline \multicolumn{5}{|l|}{ Financial difficulties } \\
\hline Baseline (T0) & $34.3 \pm 4.4$ & $21.5 \pm 4.4$ & $-12.9(0.1)$ & \\
\hline 0.5 -year follow-up $(\mathrm{T} 1)^{\mathrm{f}}$ & $34.3 \pm 5.0$ & $18.2 \pm 4.0$ & $-16.2(0.1)$ & $-19.8(0.01)$ \\
\hline 1-year follow-up (T2) & $27.6 \pm 4.2$ & $12.8 \pm 3.7$ & $-14.7(0.05)$ & $-18.0(0.004)$ \\
\hline
\end{tabular}

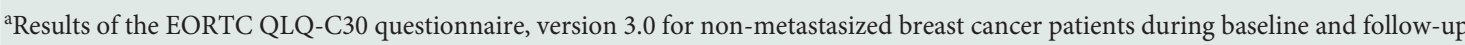
(mean \pm SE) and the association between symptoms and ECLR.

${ }^{b}$ Worsening of symptom with UICC stage IIIA $(\beta=28.2, \mathrm{p}=0.03)$.

'Improvement of symptom with VA treatment $(\beta=-13.8, \mathrm{p}=0.01)$.

${ }^{\mathrm{d}}$ Improvement of symptom with hormonal therapy $(\beta=-10.3, \mathrm{p}=0.02)$, worsening of symptom with immunological therapy $(\beta=$ $30.9, \mathrm{p}=0.001)$.

${ }^{\mathrm{e}}$ Improvement of symptom with hormonal therapy $(\beta=-10.8, \mathrm{p}=0.03)$.

f,g Improvement of symptom with age at ${ }^{\mathrm{f}} \mathrm{T} 1(\beta=-0.9, \mathrm{p}=0.008)$ and ${ }^{\mathrm{g}} \mathrm{T} 2(\beta=-0.7, \mathrm{p}=0.009)$.

${ }^{*} \mathrm{p}$-value for difference between control and treatment group (Wilcoxon-Mann-Whitney test).

${ }^{*}$ Negative $\beta$-values indicate an association with improvement while positive $\beta$-values indicate an association with worsening of symptoms.

${ }^{* * *} \mathrm{p}$-value for an association between ECLR and patient-reported outcome (multiple linear regression analysis).

ECLR = Elaborate consultations and life review; SE = standard error; EORTC = European Organisation for Research and Treatment of Cancer; QLQ = quality-of-life questionnaire; UICC = Union for International Cancer Control; VA = Viscum album L.

$p=0.002)$. Similar significant differences were observed for physical functioning at T1 (Ctrl: 66.2 \pm 5.5 , ECLR: $90.4 \pm 4.1 ; \mathrm{p}=0.008)$, role functioning (Ctrl: $51.8 \pm 6.7$, ECLR: $83.3 \pm 7.9 ; \mathrm{p}=0.001$ ), and global health status/QoL at T1 (Ctrl: $53.7 \pm 3.9$, ECLR: $79.6 \pm 5.1$; $\mathrm{p}=0.001)$, where the self-reported burden due to the chemotherapeutic regimen was significantly higher in the control group but not in the ECLR group. 
Table 5. Differences in patient-reported outcomes (functioning) between groups and association with ECLR $^{\mathrm{a}}$

\begin{tabular}{|c|c|c|c|c|}
\hline \multirow[t]{2}{*}{ QoL or function } & \multicolumn{2}{|c|}{ Questionnaire score (mean \pm SE) } & \multirow[b]{2}{*}{ Difference $\left(\mathrm{p}^{*}\right)$} & \multirow[b]{2}{*}{$\begin{array}{l}\text { Estimate }\left(\beta^{* *}\right) \\
\text { for } \operatorname{ECLR}\left(\mathrm{p}^{* * *}\right)\end{array}$} \\
\hline & Control & $\begin{array}{l}\text { ECLR treatment } \\
\text { group }\end{array}$ & & \\
\hline \multicolumn{5}{|l|}{ Global health status/QoL } \\
\hline Baseline (T0) & $57.5 \pm 2.2$ & $64.7 \pm 2.2$ & $7.2(0.1)$ & \\
\hline 0.5 -year follow-up (T1) & $63.9 \pm 2.3$ & $69.4 \pm 3.5$ & $5.5(0.2)$ & $4.2(0.35)$ \\
\hline 1-year follow-up (T2) & $64.3 \pm 2.5$ & $76.0 \pm 2.8$ & $11.7(0.004)$ & $10.9(0.01)$ \\
\hline \multicolumn{5}{|l|}{ Physical functioning } \\
\hline Baseline (T0) & $76.0 \pm 2.3$ & $82.5 \pm 2.3$ & $6.5(0.1)$ & \\
\hline 0.5 -year follow-up (T1) ${ }^{\mathrm{b}}$ & $77.1 \pm 2.5$ & $84.1 \pm 2.8$ & $6.9(0.1)$ & $5.5(0.15)$ \\
\hline 1-year follow-up (T2) & $75.6 \pm 2.6$ & $83.2 \pm 2.6$ & $7.6(0.1)$ & $3.1(0.64)$ \\
\hline \multicolumn{5}{|l|}{ Role functioning } \\
\hline Baseline (T0) & $55.5 \pm 4.2$ & $64.0 \pm 4.2$ & $8.5(0.2)$ & \\
\hline 0.5 -year follow-up (T1) & $68.3 \pm 3.5$ & $72.0 \pm 4.6$ & $3.8(0.6)$ & $6.2(0.31)$ \\
\hline 1-year follow-up (T2) & $67.5 \pm 3.8$ & $74.7 \pm 4.8$ & $7.2(0.3)$ & $1.1(0.87)$ \\
\hline \multicolumn{5}{|l|}{ Emotional functioning } \\
\hline Baseline (T0) & $52.0 \pm 3.0$ & $61.0 \pm 3.1$ & $9.1(0.1)$ & \\
\hline 0.5 -year follow-up (T1) ${ }^{\mathrm{d}}$ & $61.2 \pm 3.0$ & $66.1 \pm 3.7$ & $4.9(0.3)$ & $4.1(0.45)$ \\
\hline 1-year follow-up (T2)e & $60.8 \pm 3.1$ & $74.7 \pm 3.2$ & $13.9(0.009)$ & $11.9(0.03)$ \\
\hline \multicolumn{5}{|l|}{ Cognitive functioning } \\
\hline Baseline (T0) & $72.6 \pm 3.1$ & $78.4 \pm 3.1$ & $5.8(0.2)$ & \\
\hline 0.5-year follow-up (T1) & $73.4 \pm 2.8$ & $78.5 \pm 4.0$ & $5.0(0.3)$ & $5.6(0.28)$ \\
\hline 1-year follow-up (T2) & $71.3 \pm 3.0$ & $85.4 \pm 3.1$ & $14.4(0.004)$ & $12.6(0.01)$ \\
\hline \multicolumn{5}{|l|}{ Social functioning } \\
\hline Baseline (T0) & $58.9 \pm 4.0$ & $64 \pm 4.0$ & $5.1(0.5)$ & \\
\hline 0.5 -year follow-up (T1) & $66.2 \pm 3.8$ & $78.4 \pm 3.6$ & $12.3(0.1)$ & $8.5(0.18)$ \\
\hline 1-year follow-up (T2) & $64.6 \pm 3.9$ & $80.6 \pm 3.8$ & $16.1(0.02)$ & $12.5(0.04)$ \\
\hline
\end{tabular}

a Results of the EORTC QLQ-C30 questionnaire, version 3.0 for breast cancer patients during baseline and follow-up (mean \pm SE) and the association between functioning/QoL scores and ECLR.

${ }^{b}$ Worsening of functioning score with increasing age $(\beta=-0.5, p=0.001)$.

${ }^{c}$ Worsening of functioning score with increasing age $(\beta=-0.5, p=0.003)$.

${ }^{d}$ Worsening of functioning score with UICC stage IIIA $(\beta=-30.4, p=0.02)$.

${ }^{\mathrm{e}}$ Improvement of functioning score with massage therapy $(\beta=13.2, \mathrm{p}=0.03)$.

${ }^{*} \mathrm{p}$-value for difference between control and treatment group (Wilcoxon-Mann-Whitney test).

${ }^{* *}$ Positive $\beta$-values indicate an association with improvement while negative $\beta$-values indicate an association with worsening of QoL or functioning.

${ }^{* * *}$ p-value for association between ECLR and patient-reported outcome (multiple linear regression analysis).

$\mathrm{ECLR}=$ Elaborate consultations and life review; $\mathrm{QoL}=$ quality of life; $\mathrm{SE}=$ standard error; EORTC $=$ European Organisation for Research and Treatment of Cancer; QLQ = quality-of-life questionnaire; UICC = Union for International Cancer Control.

\section{Discussion}

Studies suggest that LR interviews improve the psychosocial well-being of terminally ill cancer patients $[11,12]$. Even though already widely applied in Anthroposophic-integrative oncology, no peer-reviewed systematic research exists on the impact of ECLR on psychosocial or physical functioning of non-metastasized breast cancer patients. Our study reveals that ECLR is associated with significant improvements of pivotal HRQL fields in non-metastasized breast cancer patients. Sustained effects were found for improvements in cognitive, emotional, and social functioning, global health/QoL, and financial difficulties.

At baseline, we observed an equal distribution of the patients' demographic and clinical characteristics as well as breast cancer subtypes between the two groups. Significant improvement of sustained emotional and social functioning was found for ECLR pa- tients compared to the control. This is similar to the results of other studies that reported psychosocial improvement in terminally ill patients [11-13]. Furthermore, our results of the effects of ECLR are in line with the evident impact of psychoeducation on emotional distress and QoL [28-32]. According to a systematic review and meta-analysis covering 198 studies and 22,238 cancer patients, individual and group psychotherapy as well as psychoeducation improved emotional distress, anxiety, depression, and HRQL [28]. Psychoeducational interventions (19 studies, 3,857 patients), which were defined as 'multimethod comprehensive interventions covering health education, coping skills training, stress management, and psychological support', showed significant improvements of emotional distress, anxiety, and depression in the medium term ( $<6$ months) and improvement of HRQL at $>6$ months. This is in accordance with previous meta-analyses in this field [29-31]. Because of this 'strong evidence base' of psychotherapy 
Fig. 2. Subgroup analysis, chemotherapy-treated patients. Significant differences in global health/ HRQL, functioning, and symptom scales between patients receiving standard care (control) and patients receiving standard care plus ECLR (ECLR). Time point: $\mathrm{T} 1=6$ months after first diagnosis. ${ }^{*} \mathrm{p}<0.05,{ }^{* *} \mathrm{p}<0.01,{ }^{* * *} \mathrm{p}<0.005$; $\mathrm{p}$-values for differences between control and ECLR group. Fatigue: Lower values indicate better symptom scores. Global health/QoL, role functioning, physical functioning: Higher values indicate better functioning. Values from the box plot (median, IQR): global health/QoL (Ctrl: 50, 42-58; ECLR: 83, 67-92), role functioning (Ctrl: 50, 33-67; ECLR: 100, 67-100), fatigue (Ctrl: 61.5, 44-67, ECLR: 22, 0-33), physical functioning (Ctrl: 66.5, 48.5-87, ECLR: 100, $80-100)$. QOL $=$ quality of life; ECLR = elaborate consultations and life review.

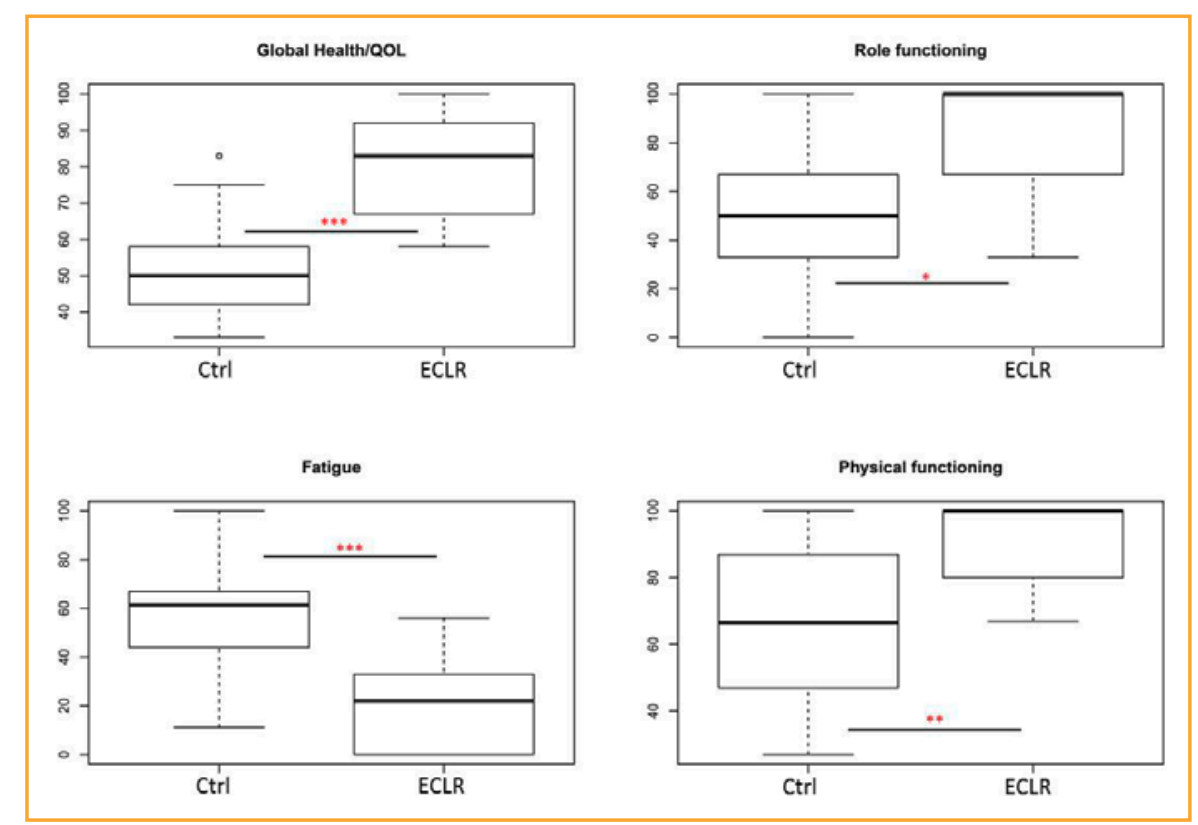

and psychoeducation [33], the recently published clinical practice guidelines on the evidence-based use of integrative therapies during and after breast cancer treatment excluded these interventions from analyses as both interventions are 'often considered to be mainstream rather than integrative or complementary'. ECLR, in form and content often viewed as part of psychoeducation, has, however, not been evaluated in meta-analyses or randomized controlled studies yet [11-13]. Therefore, little or no systematic research results and evidence have been published so far on its effect on emotional distress and HRQL. With our results we would like to contribute to fill this gap.

In the present study, improvement of short-term appetite loss and pain 6 months after diagnosis was found to be significantly associated with ECLR. Short-term appetite loss in cancer patients is mainly due to the applied oncological treatment such as chemoand/or radiotherapy [34]. An analysis of study results published between 1992 and 2008 performed by Montezari [17] revealed that, besides fatigue, pain and appetite loss were the most predominant symptomatic predictors of survival time in cancer after adjustment of demographic and clinical prognostic factors. As pain, including chronic pain, is significantly associated with HRQL in breast cancer patients, early pain management could be a determinant for the improvement of the patient's burden during cancer treatment $[35,36]$.

Our study also revealed that integrative interventions such as mistletoe therapy improved short-term appetite loss. This is in accordance with a previous randomized controlled trial investigating the effects of subcutaneous mistletoe in advanced and metastatic pancreatic cancer patients and showing an association between treatment and improvement of appetite loss during 1-12 months [37].

The results of the present study reveal a relevant negative association between anti-hormonal treatment and short- and mediumterm diarrhea. Anti-hormonal treatment significantly interferes with the estrogen homeostasis of premenopausal women by either inhibiting estrogen production or uptake through estrogen recep- tors, thus inducing alterations of gastrointestinal functions among other symptoms common in postmenopausal women [38, 39]. A prospective study revealed that a highly significant greater proportion of women in the postmenopausal state reported altered bowel function than women in the premenopausal state [40]. We conclude that anti-hormonal therapy may play a non-irrelevant role in the alterations of gastrointestinal problems of the here described breast cancer patients. Whether this may be the explanation for the negative association between diarrhea and anti-hormonal treatment in the present study has to be further elucidated.

Our data show short- and medium-term improvement of financial difficulties in non-metastasized breast cancer patients who underwent ECLR. As indicated in a 2-year prospective cohort study of 725 female patients with early breast cancer, 'financial difficulties' was the main distinguishing characteristic between patients with high stable anxiety compared to all other anxiety groups [41]. Anxiety of cancer patients who underwent ECLR could significantly be diminished in another study [12]. The present study shows that the emotional functioning could be significantly improved in the ECLR group compared to the control group. Improved emotional functioning (EORTC QLQ-C30) meant that the patient felt less tense, less irritable, less depressed and was less worried. We speculate that this improved anxiety may have had an influential effect on the patient's self-reporting of financial difficulties during the course of the study. As discussions on personal anxiety, fears, problems, and obstacles are an integral part of the ECLR, the patient could have developed new meaning and answers to his disease and also new life perspectives and may have viewed the financial problems as being less important.

In a subgroup receiving a chemotherapeutic regimen, we observed that short-term HRQL, physical functioning, and role functioning were highly significantly different between the ECLR group and the control group. More importantly, there was a marked difference in self-reported short-term fatigue in chemotherapy-treated 
patients between the two groups $(\mathrm{p}=0.002)$. Our findings resemble results of a prospective multicenter trial with 105 non-metastasized breast cancer patients, where psychoeducation as part of a multimodal program (sleep education, eurythmy therapy, painting therapy) significantly reduced fatigue/disturbed sleep at 6 months after the end of intervention [42]. We assume that ECLR may ameliorate short-term physical and role functioning difficulties as well as the fatigue burden in patients receiving chemotherapy.

Finally, in the present study, we could show an improvement of medium-term cognitive functioning in non-metastasized patients who have received ECLR. In the light of sustained (up to 11.5 years) impairment of cognitive functioning due to the chemotherapeutic regimen during primary oncological care, increasing with the intensity of chemotherapy [43], the results of our study reveal an important finding. However, we could not find this improvement of cognitive functioning in our subgroup analysis, i.e., there were no significant differences between chemotherapy-treated patients either having received ECLR or not. Perhaps this would be mainly due to the fact of comparing only small patient groups in this subgroup. Nevertheless, no final conclusion can be drawn on the medium-term effect of ECLR on chemotherapy-induced cognitive impairment at this stage.

According to Carl Roger's theory proposed in 1957, congruency of relationship, unconditional positive regard, and empathic understanding of the health professional towards the patient are 3 of 6 key aspects in commencing meaningful changes in a patient's personality $[44,45]$; these are basic principles of the ECLR in the Anthroposophic-integrative setting. The authors would like to underline the individualized and elaborate nature of the ECLR in the present study. ECLR duration was at least $30 \mathrm{~min}$ (sometimes up to $1 \mathrm{~h}$ ) and the sessions took place outside the patient's room, granting sufficient time and a discrete and adequate place for the patient's concerns and issues. ECLR sessions were repeated during the patient's hospital stay, in most cases for a second time and in some cases 3-4 times.
In 2008, a meta-analysis in breast cancer patients concluded that more qualitative research would be needed for addressing 'patient-centred solutions for evidence-based selection of optimal treatments, psychosocial interventions, patient-physician communications, allocation of resources, and indicating research priorities' [20]. Our study is one of the first to examine the influence of ECLR on non-metastasized breast cancer patients. Limitations of the study lie in the non-randomized and non-controlled nature of the design. As integrative therapies are balanced between the two compared groups, the presented results of the ECLR impact may resemble real effects in the cohort. As studies suggest that in more than $50 \%$ of postmenopausal breast cancer patients integrative therapies were applied 1 year after diagnosis, the results of our study may be extended to a greater proportion of the breast cancer population.

In conclusion, the results of the present study indicate a significant positive impact of ECLR on medium-term psychological, cognitive, and social functioning and also on the global health status/ QoL of non-metastasized breast cancer patients. Our results indicate that ECLR may have a more autonomous and important value in the context of psycho-oncological interventions in integrative oncology. In light of the previously shown impact on terminally ill patients, ECLR may be useful in ameliorating psychosocial distress not only in end-of-life care but also in earlier cancer stages.

\section{Acknowledgement}

We would like to thank all staff members at the GKH and the FIH involved in the present work.

\section{Disclosure Statement}

The authors declare no competing interests.

\section{References}

1 Butler RN: Age, death, and life review; in Doka KJ, John Breaux J, Gordon JD (eds): Living with Grief: Loss in Later Life. Washington, DC, Hospice Foundation of America, 2002, pp 3-11.

2 Butler RN: Successful aging and the role of the life review. J Am Geriatr Soc 1974;22:529-535.

3 Butler RN: Mental health and aging. Life cycle perspectives. Geriatrics 1974;29:59-60.

4 Butler RN: Successful aging. MH 1974;58:6-12.

5 Lewis MI, Butler RN: Life-review therapy. Putting memories to work in individual and group psychotherapy. Geriatrics 1974;29:165-173.

6 Quetz M: Die Bedeutung der Biographie für die anthroposophische Psychotherapie in Biographie und Krankheit; in Treichler M (Hrsg): Biographie und Krankheit. Stuttgart, Urachhaus, 1995.

7 Kienle GS, Mussler M, Fuchs D, et al: Individualized integrative cancer care in anthroposophic medicine: a qualitative study of the concepts and procedures of expert doctors. Integr Cancer Ther 2016;15:478-494.
8 Kienle GS, Albonico H-U, Baars EW, et al: Anthroposophic medicine: an integrative medical system originating in Europe. Global Adv Health Med 2013;2:20-31.

9 Colyer H: Women's experience of living with cancer. J Adv Nurs 1996;23:496-501.

10 Schad F, Thronicke A, Merkle A, et al: Implementation of an integrative oncological concept in daily care of a German certified breast cancer centre. Complement Med Res 2018;DOI: 10.1159/000478655

11 Ando M, Morita T, Okamoto T, et al: One-week shortterm life review interview can improve spiritual wellbeing of terminally ill cancer patients. Psychooncology 2008;17:885-890.

12 Ando M, Morita T, Akechi T, et al: Efficacy of shortterm life-review interviews on the spiritual well-being of terminally ill cancer patients. J Pain Symptom Manage 2010;39:993-1002.
13 Ando M, Morita T, Ahn SH, et al: International comparison study on the primary concerns of terminally ill cancer patients in short-term life review interviews among Japanese, Koreans, and Americans. Palliat Support Care 2009;7:349-355.

14 Gatta G, Mallone S, van der Zwan JM, et al: Cancer prevalence estimates in Europe at the beginning of 2000. Ann Oncol 2013;24:1660-1666.

15 Elliott J, Fallows A, Staetsky L, et al: The health and well-being of cancer survivors in the UK: findings from a population-based survey. Br J Cancer 2011;105(suppl 1):S11-S20.

16 Richards M, Corner J, Maher J: The National Cancer Survivorship Initiative: New and emerging evidence on the ongoing needs of cancer survivors. Br J Cancer 2011;105(suppl 1):S1-S4.

17 Montazeri A: Quality of life data as prognostic indicators of survival in cancer patients: an overview of the literature from 1982 to 2008. Health Qual Life Outcomes 2009;7:102. 
18 Coates AS, Hurny C, Peterson HF, et al: Quality-of-life scores predict outcome in metastatic but not early breast cancer. International Breast Cancer Study Group. J Clin Oncol 2000;18:3768-3774.

19 Efficace F, Biganzoli L, Piccart M, et al: Baseline healthrelated quality-of-life data as prognostic factors in a phase III multicentre study of women with metastatic breast cancer. Eur J Cancer 2004;40:1021-1030.

20 Montazeri A: Health-related quality of life in breast cancer patients: a bibliographic review of the literature from 1974 to 2007. J Exp Clin Cancer Res 2008;27:32.

21 Fayers P, Machin D: Quality of Life: Assessment, Analysis and Interpretation. Chichester, John Wiley \& Sons, 2000.

22 EORTC QLQ-C30 Scoring Manual. http://www.eortc. be/qol/files/SCManualQLQ-C30.pdf, 2017.

23 Schad F, Axtner J, Happe A, et al: Network Oncology (NO) - a clinical cancer register for health services research and the evaluation of integrative therapeutic interventions in anthroposophic medicine. Forsch Komplementmed 2013;20:353-360.

24 Miller AJ: Subset Selection in Regression, ed 2. New York, Chapman \& Hall/CRC, 2002.

25 Hosmer D, Lemeshow S: Applied Logistic Regression, ed 2. New York, John Wiley \& Sons, 2005.

26 Team RC: R: a language and environment for statistical computing. Vienna, R Foundation for Statistical Computing, 2016.

27 Scott NW, Fayers PM, Aaronson NK, et al: EORTC QLQ-C30 reference values. http://groups.eortc.be/qol/ sites/default/files/img/newsletter/reference_values_manual2008.pdf, 2008.

28 Faller H, Schuler M, Richard M, et al: Effects of psychooncologic interventions on emotional distress and quality of life in adult patients with cancer: systematic review and meta-analysis. J Clin Oncol 2013;31:782-793.
29 Akechi T, Okuyama T, Onishi J, et al: Psychotherapy for depression among incurable cancer patients. Cochrane Database Syst Rev 2008;(2):CD005537.

30 Luebbert K, Dahme B, Hasenbring M: The effectiveness of relaxation training in reducing treatment-related symptoms and improving emotional adjustment in acute non-surgical cancer treatment: a meta-analytical review. Psychooncology 2001;10:490-502.

31 Tatrow K, Montgomery GH: Cognitive behavioral therapy techniques for distress and pain in breast cancer patients: a meta-analysis. J Behav Med 2006;29:17-27.

32 Zimmermann T, Heinrichs N, Baucom DH: 'Does one size fit all?' Moderators in psychosocial interventions for breast cancer patients: a meta-analysis. Ann Behav Med 2007;34:225-239.

33 Greenlee H, DuPont-Reyes MJ, Balneaves LG, et al: Clinical practice guidelines on the evidence-based use of integrative therapies during and after breast cancer treatment. CA Cancer J Clin 2017;67:194-232.

34 Macquart-Moulin G, Viens P, Genre D, et al: Concomitant chemoradiotherapy for patients with nonmetastatic breast carcinoma: side effects, quality of life, and organization. Cancer 1999;85:2190-2199.

35 Laroche F, Perrot S, Medkour T, et al: Quality of life and impact of pain in women treated with aromatase inhibitors for breast cancer. A multicenter cohort study. PLoS One 2017;12:e0187165.

36 Hamood R, Hamood H, Merhasin I, et al: Chronic pain and other symptoms among breast cancer survivors: prevalence, predictors, and effects on quality of life. Breast Cancer Res Treat 2018;167:157-169.

37 Troger W, Galun D, Reif M, et al: Quality of life of patients with advanced pancreatic cancer during treatment with mistletoe: a randomized controlled trial. Dtsch Arztebl Int 2014;111:493-502, 33 p following 502.
38 Jacenik D, Cygankiewicz AI, Fichna J, et al: Estrogen signaling deregulation related with local immune response modulation in irritable bowel syndrome. $\mathrm{Mol}$ Cell Endocrinol 2017; in press. DOI: 10.1016/j.mce. 2017.07.036.

39 Tedesco FJ, Volpicelli NA, Moore FS: Estrogen- and progesterone-associated colitis: a disorder with clinical and endoscopic features mimicking Crohn's colitis. Gastrointest Endosc 1982;28:247-249.

40 Triadafilopoulos G, Finlayson M, Grellet C: Bowel dysfunction in postmenopausal women. Women Health 1998;27:55-66.

41 Saboonchi F, Petersson LM, Wennman-Larsen A, et al: Trajectories of anxiety among women with breast cancer: a proxy for adjustment from acute to transitional survivorship. J Psychosoc Oncol 2015;33:603-619.

42 Kröz M, Reif M, Glinz A, et al: Impact of a combined multimodal-aerobic and multimodal intervention compared to standard aerobic treatment in breast cancer survivors with chronic cancer-related fatigue - results of a three-armed pragmatic trial in a comprehensive cohort design. BMC Cancer 2017;17:166.

43 Stouten-Kemperman MM, de Ruiter MB, Boogerd W, et al: Very late treatment-related alterations in brain function of breast cancer survivors. J Int Neuropsychol Soc 2015;21:50-61.

44 Rogers CR: The necessary and sufficient conditions of therapeutic personality change. Psychotherapy (Chic) 2007; $44: 240-248$

45 Rogers CR: The necessary and sufficient conditions of therapeutic personality change. J Consult Psychol 1957; 21:95-103. 